\title{
Aprendizaje de lenguas mediante dispositivos móviles: una experiencia didáctica con los códigos QR
}

Mobile-assisted language learning: A teaching experience with QR codes

Elisa Sartor

Università degli Studi di Verona, Dipartimento di Lingue e Letterature Straniere

\section{Resumen}

Este trabajo se centra en una actividad de actividad y, en segundo lugar, nos propoaprendizaje de lenguas mediante dispositivos móviles llevada a cabo durante un seminario sobre enseñanza del español como lengua extranjera y tecnologías de la información y comunicación (TIC), dirigido a estudiantes de grado orientados a la carrera docente. El objetivo de esta contribución es doble: en primer lugar, pretendemos describir el marco constructivista nemos evaluar los resultados conseguidos en una encuesta anónima mediante la plataforma Formularios de Google. En las respuestas de los informantes se aprecia una actitud en pro de mejorar sus competencias digitales y utilizarlas en la enseñanza, a pesar de una situación inicial de parcial desconocimiento de las TIC y de su aplicación a la didáctica.

y los criterios que guiaron el diseño de la

Palabras clave: lingüística aplicada; constructivismo; ELE; interacción oral; TIC 


\begin{abstract}
This work focuses on a mobile-assist- activity; second, we assessed the results ed language learning exercise conducted achieved from the activity by using a blind during a seminar on teaching of Spanish survey created on Google Form. The reas a second language and information and sponses from participants showed a posicommunication technology (ICT), offered tive attitude towards improving their own to undergraduate students who may pursue digital abilities and applying them to the a career in teaching. The objective of this teaching practice, despite an initial partial contribution is two-fold: first, we intend lack of awareness about ICT and its applito describe the constructivist framework cation to didactics.
\end{abstract} and criteria that shaped the design of this

Keywords: applied linguistics; constructivism; ssL; oral interaction; ICT 


\section{Introducción}

La experiencia didáctica aquí presentada ${ }^{1}$ surgió a raíz del seminario Lengua Española: Didáctica y Nuevas Tecnologías, que se celebró en mayo de 2017 en la Universidad de Verona. Con ocasión del taller de clausura de las jornadas de estudio, decidimos planear una actividad basada en los códigos $\mathrm{QR},{ }^{2}$ concretamente una caza del tesoro por el campus universitario con pistas inspiradas en una de las ponencias del día anterior y en el temario de la asignatura oficial de Lingüística Española I.

El objetivo de este trabajo es doble: por un lado, pretendemos describir los criterios que orientaron el diseño de la actividad y, por otro, nos proponemos evaluar los resultados conseguidos a través de ella. En relación con la primera finalidad adquiere especial relevancia el marco teórico que enlaza la práctica del aprendizaje móvil a la teoría constructivista, tal como se ha ido desarrollando en años recientes gracias a la labor pionera de estudiosas como Agnes Kukulska-Hulme, Agniezska Palalas y Debra Hoven. ${ }^{3}$ Con respecto a la segunda finalidad, en cambio, adoptamos un enfoque más bien práctico y elaboramos una encuesta para medir la eficacia de la actividad en términos de propensión al aprendizaje de lenguas mediante dispositivos móviles (MALL, por sus siglas en inglés, mobile-assisted language learning) por parte de los participantes.

1 Este estudio se desarrolló en el marco de la beca de investigación "Le nuove tecnologie nella formazione degli insegnanti di lingua spagnola”, Università degli Studi di Verona, Dipartimento di Lingue e Letterature Straniere; responsable científico, profesora Francesca Dalle Pezze.

2 El código QR (quick response code, 'código de respuesta rápida') es un código de barras formado por cuadrados negros sobre fondo blanco, que permite almacenar información (normalmente breves textos o la dirección de una página web) y que se puede leer a través de un dispositivo móvil gracias a aplicaciones tanto gratuitas como de pago. El material didáctico para la actividad descrita en este informe se realizó empleando la aplicación gratuita Qrafter, compatible con móviles de la marca iPhone.

3 Para profundizar en la literatura científica sobre el aprendizaje móvil, de entre los cuantiosos ensayos publicados en la última década, remitimos especialmente a Kukulska-Hulme y Shield (2008), Hoven y Palalas (2011), Tai (2012), Palalas (2015), Burston (2016), Yates y Palalas (2016), además del volumen misceláneo editado por Palalas y Ally (2016), que recoge numerosas contribuciones tanto teóricas como descriptivas y aplicativas. 


\section{Estado de la cuestión. El aprendizaje de lenguas mediante dispositivos móviles}

La rápida y masiva difusión de la tecnología de la información y de la comunicación en la sociedad y, por consiguiente, en el ámbito educativo, ha propiciado una reflexión teórica sobre su potencial didáctico y su paulatina incorporación a la enseñanza. ${ }^{4}$ En este reporte, en concreto, pretendemos acotar la investigación al cambio de paradigma impulsado por el uso de dispositivos móviles. ${ }^{5}$

El punto de partida para un análisis en este campo tiene que ser el metaestudio llevado a cabo por Jack Burston (2016), en el que el autor toma en consideración los 570 artículos publicados entre 1994 y 2012 sobre MALL, examinando los 345 que detallan experiencias didácticas de aplicación en el marco del aprendizaje móvil. Desde sus comienzos con los diccionarios bilingües en Japón (Burston, 2016: 6), el empleo de MALL ha evolucionado hasta incluir todo tipo de actividades (comprensión lectora y auditiva, ejercicios de gramática, pronunciación y vocabulario). Sin embargo, el análisis llevado a cabo por Burston muestra que $75 \%$ de las aplicaciones examinadas en los estudios favorece el trabajo individual por parte del alumno en vez de un enfoque colaborativo; más aún, $85 \%$ de los casos considerados promueven un modelo centrado en el profesor y solo $15 \%$ centrado en el alumno (2016: 18). El autor subraya la discrepancia entre los enfoques teóricos vigentes en las últimas dos décadas, especialmente el modelo constructivista y la permanencia de una orientación estructuralista en casi la totalidad de las aplicaciones MALL (2016: 24).

Desde luego, esta contradicción ya había sido destacada por Agnes Kukulska-Hulme y Lesley Shield en un ensayo de 2008, en el que, describiendo las diferencias entre las aplicaciones educativas para ordenadores (compu-

4 En lo que se refiere a la educación secundaria en Italia, por ejemplo, las competencias digitales son una de las tres prioridades estratégicas señaladas por el ministerio competente para la formación del profesorado en el trienio 2016/19 (Ministero dell'Istruzione, dell'Università e della Ricerca [Ministerio de Educación,Universidad e Investigación de Italia], 2016: 26).

5 Para la definición de dispositivos móviles seguimos a la UNESCO: "son digitales, portátiles, controlados por lo general por una persona (y no por una institución), que es además su dueña, tienen acceso a Internet y capacidad multimedia, y pueden facilitar un gran número de tareas, especialmente las relacionadas con la comunicación” (2013: 6). 
ter-assisted language learning, o sea, CALL) y la nueva generación de MALL, las dos autoras afirman: "until relatively recently, MALL activities rather mirrored early CALL activities where electronic quizzes, grammar drills and vocabulary lists dominated" (Kukulska-Hulme \& Shield, 2008: 283). En cambio, las actividades MALL "encourage collaboration and co-construction of knowledge; learners [have] to find information and share it with their peers in order to build up an overall understanding of a real-world problem"7 (Kukulska-Hulme \& Shield, 2008: 280).

Agniezska Palalas y Debra Hoven (2016) también abogan por un enfoque centrado en el alumno, y en un trabajo reciente recalcan la importancia de aprovechar el potencial comunicativo ofrecido por los dispositivos móviles, recordando, además, que mobile learning no significa simplemente "aprendizaje a través de dispositivos móviles", sino más bien "the experience and outcome that is enabled by any technology or interplay of technologies that afford mobile learners with the construction of knowledge or acquisition of skills across various contexts. [MALL] is enriched by social interaction"8 (Palalas \& Hoven, 2016: 50-51). "Construcción de conocimientos" es la palabra clave que remite a una perspectiva constructivista, modelo en el que debería basarse la pedagogía MALL, como señalan las autoras:

While traditional models of language teaching were anchored in the transmission-oriented paradigm, newer models are being shaped by constructivist theories of learning and more participatory student-centered approaches to teaching ${ }^{9}$ (Palalas \& Hoven, 2016: 70).

6 "Hasta una época bastante reciente las actividades MALL reproducían las primeras actividades CALL, entre las que prevalecían exámenes en formato electrónico, ejercicios de gramática y listas de vocabulario" (las traducciones de todas las citas son nuestras).

7 "Fomentan la cooperación y la construcción colaborativa del conocimiento; los aprendices tienen que encontrar información y compartirla con sus pares para desarrollar una comprensión global de un problema del mundo real."

8 "La experiencia y el resultado posibilitados por cualquier tecnología o interacción entre tecnologías que permita a los aprendices móviles la construcción del conocimiento o la adquisición de competencias en varios contextos. [El aprendizaje móvil] es enriquecido por la interacción social."

9 "Los modelos tradicionales de enseñanza de lenguas estaban anclados en paradigmas orientados a la transmisión [de conocimientos], mientras que los modelos más recientes son moldea- 
La interconexión entre teoría y práctica - y especialmente entre el marco constructivista y MALL - se ha trazado en un reciente ensayo de Nicholas Yates y Agniezska Palalas (2016), en el que se sientan las bases para la creación de actividades MALL que, en la opinión de los autores, deberían vertebrarse según cuatro principios: "Make learning authentic; Connect resources to theory; Learn by doing; Balance individual and social learning"10 (Yates \& Palalas, 2016: 184). Y justamente estas son las directrices en las que nos hemos inspirado a la hora de planear nuestra actividad de caza del tesoro, con algunas modificaciones, como veremos, debido al contexto en el que íbamos a presentar la actividad.

\section{Diseño de la actividad MALL}

Para empezar, hay que recordar que esta actividad no se elaboró para una clase de lengua, sino para proporcionar una muestra de MALL en un seminario sobre didáctica de español como lengua extranjera (ELE), de ahí su brevedad y la ausencia de tareas introductorias y/o de producción activa por parte de los alumnos.

Al planear la actividad seguimos las indicaciones ofrecidas por Yates y Palalas (2016: 183) comentadas supra: en primer lugar, en vez de explicar qué se entiende por MALL, decidimos emplear el modelo learn by doing, involucrando directamente a los participantes del seminario en una práctica de MALL, esto es, una caza del tesoro ${ }^{11}$ con códigos QR. En segundo lugar, nos pareció importante utilizar materiales auténticos, por ejemplo, un video de YouTube, además de contenidos extraídos del temario de la asignatura oficial de Lingüística Española I que los participantes, en caso de dudas, podrían comprobar desde su cuenta en la plataforma Moodle de la universidad. En tercer lugar, diseñamos la tarea según un marco constructivista, ofreciendo andamios cognitivos suficientes (pistas, acceso a internet y un hablante nativo por cada equipo de traba-

dos por las teorías constructivistas del aprendizaje y por un enfoque más participativo orientado al alumno en relación con la enseñanza."

10 "Transformar el aprendizaje en algo auténtico; relacionar los recursos con la teoría; aprender haciendo; encontrar un equilibrio entre el aprendizaje individual y el aprendizaje social."

${ }^{11}$ La actividad de caza del tesoro se basa en pistas contenidas en códigos QR, que se escanean y se leen a través de dispositivos móviles. En el Anexo A se proporciona la transcripción de las pistas y en el Anexo B un ejemplo de códigos QR elaborados para esta actividad. 
jo) para que los participantes pudieran desempeñar la tarea de manera autónoma debatiendo y negociando opciones, comunicando constantemente en L2 y, al final, colaborando en la construcción del significado global de la actividad. Por último, no fue posible, debido a la limitada extensión de la tarea, buscar un equilibrio entre aprendizaje individual y social; lo que planteamos, en cambio, fue el dinamismo entre colaboración (dentro del mismo equipo) y la competencia (entre los dos equipos).

\subsection{Los participantes}

La actividad iba dirigida a alumnos de la Universidad de Verona matriculados en el segundo o el tercer curso del grado de Lengua y Literatura Extranjeras. En concreto, las seis alumnas que participaron en el seminario Lengua Española: Didáctica y Nuevas Tecnologías cursaban el currículo de estudios literarios-filológicos y tenían un nivel B2-C1 de lengua española. Durante el seminario manifestaron su intención de trabajar en el ámbito de la enseñanza de las lenguas modernas.

El perfil académico de las alumnas fue decisivo a la hora de elaborar los contenidos de la caza del tesoro, dado que era nuestra intención redactar pistas relacionadas con el tema del seminario, esto es, la lengua española. A falta de información específica sobre los materiales que los ponentes iban a utilizar (excepto el video de YouTube, adelantado por la profesora Alejandra Ulla en un intercambio de correos electrónicos), decidimos articular las preguntas alrededor del temario de la asignatura Lingüística Española I, que todos los participantes habían estudiado en el primer curso por ser una asignatura obligatoria del currículo filológico.

\subsection{Objetivo y método}

El objetivo de la actividad es el de fomentar la interacción oral en L2 entre los participantes durante la realización de la tarea común, que consiste en solucionar una serie de pistas relacionadas con contenidos impartidos durante el seminario mismo y la asignatura oficial de Lingüística Española I. Con este fin se dividió a las alumnas en dos grupos para maximizar la oportunidad de intercambios orales 
en L2 durante la actividad y se incorporó a un hablante nativo en cada equipo ${ }^{12}$ para asegurar el uso del español como única lengua vehicular en todas las comunicaciones relativas a la tarea. Luego, se diseñó la actividad de manera que los dos grupos compitieran entre sí para completar la tarea rápidamente, logrando, por lo tanto, un equilibrio entre colaboración intragrupal y competición intergrupal.

\subsection{Procedimiento}

Durante la preparación de la tarea se cuidó especialmente la elaboración de las pistas, que iban a ser las mismas para los dos equipos, con el objetivo de garantizar condiciones equitativas a los participantes. La diferencia, sin embargo, residía en la dirección de la carrera que cada equipo tendría que recorrer para evitar que los dos marcharan en paralelo. De ahí que un equipo trabajara con códigos QR enmarcados en paspartú amarillo (véase Anexo B, Figura 5) y el otro en paspartú verde (véase Anexo B, Figura 6). Al principio de la actividad se entregó un sobre del color correspondiente a cada equipo, que contenía una pregunta idéntica sobre los diccionarios del siglo XVII con dos desenlaces posibles. Para simplificar la preparación de la actividad, se decidió que las respuestas incorrectas remitirían a un "callejón sin salida", esto es, un lugar desprovisto de ulteriores pistas. En cambio, las respuestas correctas llevarían a los participantes a tres sitios (cafetería, despacho del coorganizador del seminario y biblioteca del departamento ${ }^{13}$ pero en orden distinto según el color de las tarjetas.

Al final de la actividad, los dos grupos convergerían en la biblioteca del departamento, donde encontrarían la última pista: "Mirad otra vez este video: https://www.youtube.com/watch?v=bZb6So6d9cE. Fijaos en el título de una de las obras nombradas en él y sacad un ejemplar de la biblioteca. ¡Habéis encon-

${ }^{12}$ Respectivamente, el profesor Román Navarro y la profesora Alejandra Ulla Lorenzo, ponentes en el seminario organizado por el Departamento, pero procedentes de otros centros educativos; decidimos involucrarlos en la actividad justamente por no ser profesores en las asignaturas oficiales de la Universidad de Verona, con el objetivo de que los participantes en la caza del tesoro pudieran interactuar de forma espontánea en L2 sin tener miedo a ser evaluados por los docentes de Verona.

${ }^{13}$ A este respecto, cabe subrayar la importancia de avisar previamente a los empleados de la cafetería, la biblioteca, etc., de que una actividad de este tipo va a tener lugar en las instalaciones y sus alrededores. 
trado el tesoro! Llevad el libro al aula del seminario para recibir el premio". El enlace remitía a un breve documental acerca del Fondo Antiguo de la Biblioteca Nacional de España, que la profesora Ulla había incorporado a su presentación del día anterior sobre repertorios bibliográficos. Claro está que, en las fases de preparación de la actividad, comprobamos que en la biblioteca de departamento había ejemplares de los libros mencionados en el video y que, sobre todo, se podían solicitar a préstamo. Lo que no se pudo controlar, en cambio, fue el simple hecho de que la bibliotecaria no estaba en el mostrador al llegar el segundo equipo, de ahí que este consiguiera completar la tarea con bastante retraso con respecto al primer equipo.

\subsection{Análisis}

La actividad se evaluó a través de una encuesta realizada en la plataforma Formularios de Google, que permitía respetar el anonimato de los participantes. Desafortunadamente, solo cinco de las seis alumnas aceptaron tomar parte en el sondeo.

La encuesta se articuló en dos partes, la primera atañía a cuestiones generales sobre el seminario y la segunda hacía hincapié en TIC. En ambas los participantes tenían que valorar una afirmación con una puntuación de 1 (totalmente en desacuerdo) a 5 (totalmente de acuerdo). En los dos gráficos de barra reproducidos a continuación se presentan las respuestas de las alumnas a las preguntas más significativas de la encuesta en relación con su perfil académico (Figura 1) y con sus competencias digitales (Figura 2).

Los dos gráficos de pastel recogen, en cambio, la valoración de las TIC en la enseñanza por parte de las alumnas (Figura 3) y su interés por herramientas digitales específicas (Figura 4).

Las respuestas de la primera sección de la encuesta (Figura 1) confirman el perfil de los participantes, puesto que $100 \%$ de las alumnas declara tener un interés por la didáctica de las lenguas extranjeras y trabaja o quiere trabajar en el ámbito de la enseñanza (puntuación de 3 a 5); solo 20\% de ellas no tenían conocimientos previos suficientes alrededor de los temas tratados durante el seminario. Este último dato cambia netamente en relación con las TIC (Figura 2), ya que apenas $40 \%$ alega conocimientos previos y, antes de estas jornadas de estudio, $60 \%$ no sabía que las nuevas tecnologías se pueden aplicar a la didáctica. 

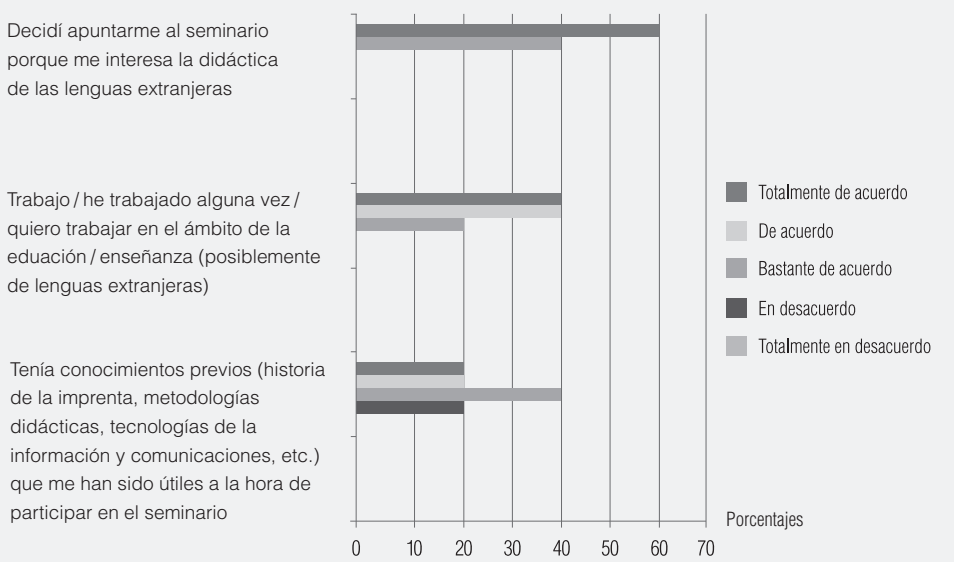

Figura 1. Datos elaborados a partir de la sección de la encuesta relacionada con el perfil de los participantes
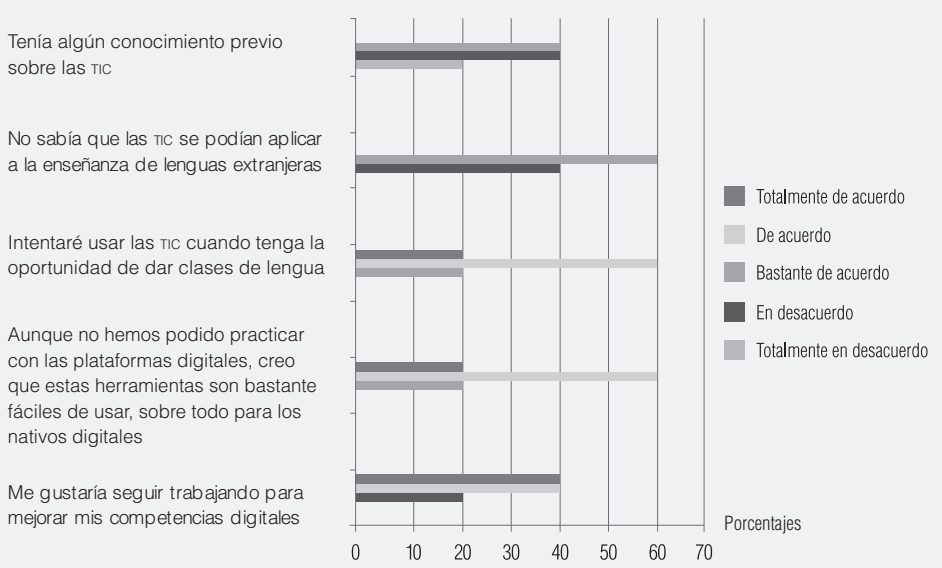

Figura 2. Datos elaborados a partir de la sección de la encuesta relacionada con las competencias digitales de los participantes

Sin embargo, la encuesta demuestra una tendencia positiva (Figura 2), ya que $100 \%$ cree que las TIC se pueden manejar con relativa facilidad y piensa emplearlas cuando tenga la oportunidad de dar clases de lengua; solo $20 \%$ no manifiesta la intención de trabajar para mejorar sus competencias digitales. $80 \%$ 


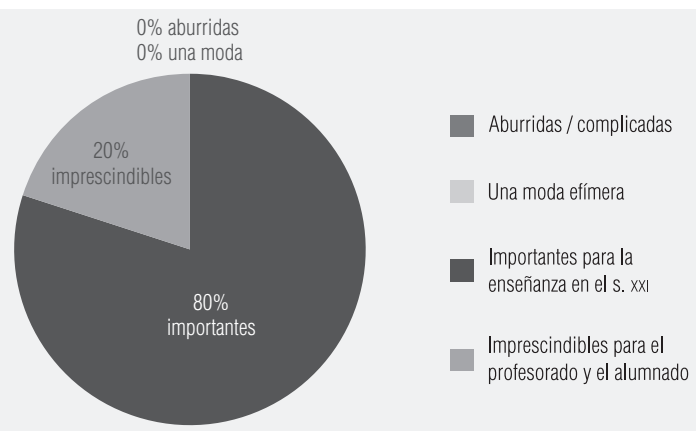

FIGURA 3. Datos elaborados a partir de la pregunta de opción múltiple "Las TIC me parecen..."

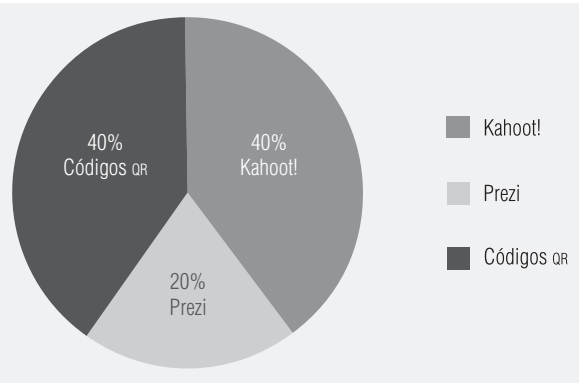

Figura 4. Datos elaborados a partir de la pregunta de opción múltiple "La herramienta que más me ha interesado/parecido útil es..."

opina que las TIC son importantes en la didáctica actual y $20 \%$ que estas son imprescindibles tanto para el profesorado como para el alumnado (Figura 3). En cuanto al interés despertado, de las herramientas con las que los participantes pudieron practicar, Kahoot! y los códigos QR empatan con un 40\%, mientras que Prezi recoge un 20\% de preferencias (Figura 4).

\section{Evaluación de la actividad}

En el marco del seminario sobre didáctica y nuevas tecnologías, la caza del tesoro con códigos QR ha sido un ejemplo adecuado de MALL: por un lado, refleja los cuatro principios mencionados supra: "Make learning authentic; Connect resources 
to theory; Learn by doing; Balance individual and social learning" (Yates \& Palalas, 2016: 184); por otro, a través de la caza del tesoro se ha conseguido ilustrar el potencial de las TIC en el aula y, pese a la necesaria brevedad de la actividad, la retroalimentación ha sido muy favorable. Las respuestas de los informantes destacan una actitud en pro de mejorar las competencias digitales (80\%) y utilizarlas en la enseñanza (100\%), frente a una situación de partida inicial de desconocimiento de las propias Tic (60\%) y de sus aplicaciones en la didáctica (60\%).

\section{Conclusiones}

Esta nota recoge la descripción de una experiencia didáctica con MALL que se llevó a cabo durante un seminario sobre nuevas tecnologías y didáctica en la Universidad de Verona y que no formaba parte de una clase de lengua, sino que representaba una muestra del potencial de MALL dirigida a futuros docentes de ELE.

De acuerdo con la más reciente literatura científica, se planteó la actividad según el marco constructivista, esto es, se diseñó de tal manera que los dispositivos móviles fomentaran la colaboración entre los alumnos para la elaboración, entre todos, del significado global de la tarea. La eficacia de la actividad se evaluó a través de una encuesta anónima que tomaba en consideración tanto el nivel de conocimiento (y la percepción) de las TIC por parte de los informantes como su disposición a mejorar las propias competencias digitales y a emplear las nuevas tecnologías en la práctica docente.

La retroalimentación proporcionada por los participantes ha sido muy favorable, sin embargo, cabe subrayar el límite de esta experiencia: tratándose de un seminario optativo, la participación en la actividad fue bastante baja, apenas seis alumnas, de la cuales cinco aceptaron tomar parte en la encuesta. De ahí que la información y los datos recogidos sean parciales y necesiten corroborarse por un estudio más abarcador en términos de duración y de número de alumnos implicados.

En conclusión, se ha realizado un primer acercamiento a la didáctica MALL en el ámbito de la enseñanza ELE en un contexto determinado (Universidad de Verona) y en el marco de un proyecto de investigación sobre nuevas tecnologías aplicadas a la didáctica. Lo que se necesita ahora es validar algunos de los resultados que se han destacado en este trabajo — por ejemplo la actitud positiva de los participantes hacia las actividades MALL, la percepción de la asequibilidad 
de las competencias digitales y la disposición a implicarse en su desarrollo- en una escala más amplia y a largo plazo.

\section{Referencias}

Burston, JACK (2016). The reality of MALl: Still on the fringes. En Agnieszka Palalas \& Mohamed Ally (Eds.), The international handbook of mobile-assisted language learning (pp. 2-43). Beijing: China Central Radio \& Tv University Press. Recuperado de https://www.academia.edu/27892480/ The_International_Handbook_of_Mobile-Assisted_Language_Learning

Hoven, Debra, \& Palalas, Agnieszka (2011). (Re)Conceptualizing design approaches for mobile language learning. CALICO Journal, 28(3), 699-720. Recuperado de https://auspace.athabascau.ca/bitstream/handle/2149/3167/Proofs_Hoven\%26Palalas.pdf?sequence=1\&isAllowed=y

Kukulska-Hulme, Agnes, \& Shield, Lesley (2008). An overview of mobile assisted language learning: From content delivery to supported collaboration and interaction. ReCALL, 20(3), 271-289. Recuperado de https://www.researchgate.net/publication/42795774_An_Overview_of_Mobile_Assisted_Language_Learning_From_Content_Delivery_to_Supported_ Collaboration_and_Interaction

Ministero dell'IstruZione, Dell'Università e della Ricerca [Ministerio de EduCaCión, Universidad e InVestigación de Italia] (MiUr) (2016). Piano per la formazione dei docenti 2016-2019 [Plan para la formación del profesorado en el trienio 2016-2019]. Promulgado el 19.10.2016. Recuperado de http://www.istruzione.it/allegati/2016/Piano_Formazione_3ott.pdf

Palalas, AgnieszKa (2015). The ecological perspective on the "anytime anyplace" of mobile-assisted language learning. En Elżbieta Gajek (Ed.), Technologie mobilne w ksztatceniu językowym [Mobile technologies in foreign language teaching] (pp. 29-48). Varsovia: Texter. Recuperado de http://www.academia.edu/20270042/Palalas_A._2015_-_The_Ecological_Perspective_on_the_Anytime_Anyplace_of_Mobile-Assisted_Language_Learning

Palalas, Agnieszka, \& Ally, Mohamed (Eds.) (2016). The international handbook of mobile-assisted language learning. Beijing: China Central Radio \& TV University Press. Recuperado de https://www.academia.edu/27892480/The_International_Handbook_of_Mobile-Assisted_ Language_Learning

Palalas, Agnieszka, \& Hoven, Debra (2016). Emerging pedagogies for Mall. En Agnieszka Palalas \& Mohamed Ally (Eds.), The international handbook of mobile-assisted language learning (pp. 44-85). Beijing: China Central Radio \& TV University Press. Recuperado de 
https://www.academia.edu/27892480/The_International_Handbook_of_Mobile-Assisted_ Language_Learning

TAI, YAming (2012). Contextualizing a MALl: Practice design and evaluation. Educational Technology \& Society, 15(2), 220-230.

UNESCO (Organización de las Naciones Unidas para la Educación, la Ciencia y la Cultura) (2013). Directrices de la UNESCO para las políticas de aprendizaje móvil. París: Organización de las Naciones Unidas para la Educación, la Ciencia y la Cultura. Recuperado de http://unesdoc. unesco.org/images/0021/002196/219662S.pdf

Yates, Nicholas, \& Palalas, Agnieszka (2016). Principles and strategies to design resources for mobile-assisted language learning environments. En Agnieszka Palalas \& Mohamed Ally (Eds.), The international handbook of mobile-assisted language learning (pp. 180-220). Beijing: China Central Radio \& TV University Press. Recuperado de https://www.academia.edu/27892480/ The_International_Handbook_of_Mobile-Assisted_Language_Learning 


\section{Anexo A}

Pistas elaboradas para la actividad de caza del tesoro. La letra A corresponde a las pistas enmarcadas en paspartú amarillo y la letra в a las pistas enmarcadas en paspartú verde.

Pista 1. Se entregó a los equipos en un sobre.

Vamos a empezar con una pregunta sobre los diccionarios del siglo XVII. ¿Quién es el autor del Tesoro de la lengua castellana o española (Madrid, 1611)?

A

Si es Sebastián de Covarrubias Horozco id a la cafetería del comedor, si es Alfonso de Palencia id a la oficina cus.

B

Si es Sebastián de Covarrubias Horozco id al despacho del prof. De Beni, si es Alfonso de Palencia id a la oficina ASE ESN.

Pista 2. Se encontraba en la cafetería para el equipo a y en el despacho del profesor De Beni para el в.

Los incunables son:

A

- Libros anteriores a la invención de los tipos móviles por Gutenberg > id a la oficina ASE ESN.

- Libros impresos durante el siglo Xv > id al despacho del prof. De Beni.

B

- Libros anteriores a la invención de los tipos móviles por Gutenberg > id a la oficina cus.

- Libros impresos durante el siglo xv > id a la cafetería del comedor.

Pista 3. Se encontraba en el despacho del profesor De Beni para el equipo a y en la cafetería para el в. 
Cuando bien comigo pienso mui esclarecida Reina: y pongo delante los ojos el antigüedad de todas las cosas: que para nuestra recordación e memoria quedaron escriptas: una cosa hallo y saco por conclusión mui cierta: que siempre la lengua fue compañera del imperio: y de tal manera lo siguió: que junta mente començaron. crecieron. y florecieron. y después junta fue la caída de entrambos.

A

Si pensáis que el autor de estas célebres líneas es Antonio de Nebrija id a la biblioteca de "Romanistica"; si el autor es Gonzalo Correas bajad al cLA.

B

Si pensáis que el autor de estas célebres líneas es Antonio de Nebrija id a la biblioteca de "Romanistica"; si el autor es Gonzalo Correas id al Aula Magna.

Pista 4. Se encontraba en la biblioteca para ambos equipos.

\section{A y B}

Mirad otra vez este video: https://www.youtube.com/watch?v=bZb6So6d9cE

Fijaos en el título de una de las obras mencionadas en él y sacad un ejemplar de la biblioteca. ¡Habéis encontrado el tesoro! Llevad el libro al aula del seminario para recibir el premio.

\section{Anexo B}

Códigos QR realizados para la actividad de caza del tesoro: la pista 2a (paspartú amari1lo) y la pista $3 b$ (paspartú verde).

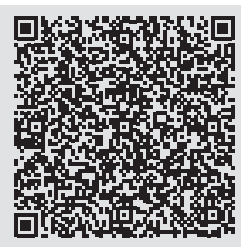

FIgURA 5. Código QR de la pista 2a

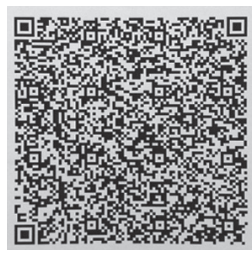

FIgura 6. Código QR de la pista $3 \mathrm{~b}$ 